\title{
Invisible widths of heavy mesons
}

\author{
Bhubanjyoti Bhattacharya, ${ }^{1}$ Cody M. Grant, ${ }^{2}$ and Alexey A. Petrov ${ }^{2}$ \\ ${ }^{1}$ Department of Natural Sciences Lawrence Technological University, Southfield, Michigan 48075, USA \\ ${ }^{2}$ Department of Physics and Astronomy Wayne State University, Detroit, Michigan 48201, USA
}

(Received 10 October 2018; published 24 May 2019)

\begin{abstract}
We revisit calculations of invisible widths of heavy mesons in the standard model, which serve as benchmarks for the studies of production of light, long-lived neutral particles in heavy meson decays. We challenge the common assumption that in the standard model these widths are dominated by meson decays into a two-neutrino final state and prove that they are dominated by decays into four-neutrino final states. We show that current estimates of the invisible widths of heavy mesons in the standard model underestimate the effect by orders of magnitude. We examine currently available experimental data on invisible widths and place constraints on the properties of dark photons. We also comment on the invisible widths of the kaons.
\end{abstract}

DOI: 10.1103/PhysRevD.99.093010

Experimental studies of light, $m \sim \mathcal{O}\left(0.1-10^{3}\right) \mathrm{MeV}$, weakly interacting long-lived particles (WILLPs) have received considerable attention recently, in part due to development of new models of dark matter (DM). These particles could help resolve several problems in physics of dark matter, both by being DM states and/or serving as mediators between visible and dark sectors of our Universe [1]. As such, extensive experimental programs of searches for light WILLPs [2-5] have been put forward at several experimental centers around the world. For recent constraints on candidates for the light particles such as axionlike states or dark photons see $[6,7]$.

If these WILLP states exist, they could also be produced in the decays of mesons, such as $B, D$, or even $K$. One of the tantalizing experimental signatures of such transitions includes "invisible" decays of heavy meson states [8], as light WILLPs do not interact with the detectors. Currently operating experiments Belle II and BESIII at $e^{+} e^{-}$machines in Japan and China, as well as experiments at future flavor factories, are the ideal places for experimental studies of such decays. This is because flavor factories operate at the $\Upsilon(4 S)$ $(b \bar{b})$ or $\psi(3770)(c \bar{c})$ resonances that decay into a correlated state of $B_{d}^{0}\left(D^{0}\right)$ meson pairs. Thus, invisible $B_{d}^{0}\left(D^{0}\right)$ decays into WILLPs can be tagged with nonleptonic decays of $\bar{B}_{d}^{0}\left(\bar{D}^{0}\right)$ decays "on the other side."

Current experimental constraints on those decays come from the analyses done at $B A B A R$ and Belle collaborations

Published by the American Physical Society under the terms of the Creative Commons Attribution 4.0 International license. Further distribution of this work must maintain attribution to the author(s) and the published article's title, journal citation, and DOI. Funded by SCOAP ${ }^{3}$. (for $B^{0}$ ) and by Belle collaboration (for $D^{0}$ ). No signals have been observed so far, so upper limits on the branching fractions of heavy mesons decaying to invisible final states $\mathcal{B}\left(B_{d}^{0} \rightarrow \not E\right)<1.3 \times 10^{-4}$ (Belle) [9] and $\mathcal{B}\left(B_{d}^{0} \rightarrow \mathscr{E}\right)<$ $2.4 \times 10^{-5}(B A B A R)[10]$ for the $b$-flavored mesons and $\mathcal{B}\left(D^{0} \rightarrow \not E\right)<9.4 \times 10^{-5}$ (Belle) [11] for charmed mesons have been set at $90 \%$ confidence level.

If measurements of invisible width of a heavy meson are to be used in placing constraints on new physics models [8], standard model (SM) backgrounds to those modes need to be estimated. While different experiments have different experiment-specific backgrounds for such processes related to "missing" particles in their detectors [9-11] that can be controlled with various experimental methods, the irreducible SM backgrounds to invisible meson decays have not received complete attention in the theoretical literature.

The only irreducible SM background that has the same experimental signature is heavy meson decays into the final states containing only neutrinos. Transitions of a $B_{q}^{0}\left(D^{0}\right)$ meson into such final states are described by an effective Lagrangian,

$$
\begin{aligned}
\mathcal{L}_{\text {eff }}= & -\frac{4 G_{F}}{\sqrt{2}} \frac{\alpha}{2 \pi \sin ^{2} \theta_{W}} \\
& \times \sum_{l=e, \mu, \tau} \sum_{k} \lambda_{k} X^{l}\left(x_{k}\right)\left(J_{Q q}^{\mu}\right)\left(\bar{\nu}_{L}^{l} \gamma_{\mu} \nu_{L}^{l}\right),
\end{aligned}
$$

where $J_{Q q}^{\mu}=\bar{q}_{L} \gamma^{\mu} b_{L}$ for beauty, and $J_{Q q}^{\mu}=\bar{u}_{L} \gamma^{\mu} c_{L}$ for charm transitions, and we consider Dirac neutrinos. The functions $\lambda_{k} X^{l}\left(x_{k}\right)$ are combinations of the CabbiboKobayashi-Maskawa factors and Inami-Lim functions. These functions are dominated by the top-quark contribution for $b \rightarrow q$ transitions, so 


$$
\sum_{k} \lambda_{k} X^{l}\left(x_{k}\right)=V_{t q}^{*} V_{t b} X\left(x_{t}\right)
$$

where $x_{t}=m_{t}^{2} / M_{W}^{2}$ and

$$
X\left(x_{t}\right)=\frac{x_{t}}{8}\left[\frac{x_{t}+2}{x_{t}-1}+\frac{3\left(x_{t}-2\right)}{\left(x_{t}-1\right)^{2}} \ln x_{t}\right] .
$$

Perturbative QCD corrections [12] would numerically change Eq. (3) by at most 10\%, and therefore are neglected. For charm $c \rightarrow u$ transitions we keep the contributions from both internal $b$ and $s$-quarks,

$$
\sum_{k} \lambda_{k} X^{l}\left(x_{k}\right)=V_{c s}^{*} V_{u s} X^{l}\left(x_{s}\right)+V_{c b}^{*} V_{u b} X^{l}\left(x_{b}\right),
$$

where $X^{l}\left(x_{q}\right)=\bar{D}\left(x_{q}, y_{l}\right) / 2$ with $y_{l}=m_{l}^{2} / m_{W}^{2}$ are related to the Inami-Lim functions [13],

$$
\begin{aligned}
\bar{D}\left(x_{q}, y_{l}\right)= & \frac{1}{8} \frac{x_{q} y_{l}}{x_{q}-y_{l}}\left(\frac{y_{l}-4}{y_{l}-1}\right)^{2} \ln y_{l} \\
& +\frac{x_{q}}{8}\left[\frac{x_{q}}{y_{l}-x_{q}}\left(\frac{x_{q}-4}{x_{q}-1}\right)^{2}+1+\frac{3}{\left(x_{q}-1\right)^{2}}\right] \ln x_{q} \\
& +\frac{x_{q}}{4}-\frac{3}{8}\left(1+3 \frac{1}{y_{l}-1}\right) \frac{x_{q}}{x_{q}-1} .
\end{aligned}
$$

Given this, one can easily estimate branching ratios for $B_{q}(D) \rightarrow \nu \bar{\nu}$ decays. One can immediately notice that the left-handed structure of the Lagrangian results in helicity suppression of these decays due to the fact that initial state is a spin-0 meson. The branching ratio is

$$
\mathcal{B}\left(B_{q} \rightarrow \nu \bar{\nu}\right)=\frac{G_{F}^{2} \alpha^{2} f_{B_{q}}^{2} M_{B_{q}}^{3}}{16 \pi^{3} \sin ^{4} \theta_{W} \Gamma_{B_{q}}}\left|V_{t b} V_{t q}^{*}\right|^{2} X\left(x_{t}\right)^{2} x_{\nu}^{2},
$$

where $x_{\nu}=m_{\nu} / M_{B_{q}}$ and $\Gamma_{B_{q}}=1 / \tau_{B_{q}}$ is the total width of the $B_{q}$ meson. We also summed over all possible neutrino states, i.e., $\nu \bar{\nu}=\sum_{i=1}^{3} \nu_{i} \bar{\nu}_{i}$.

As can be seen from Eq. (6), the branching ratio is exactly 0 in the minimal standard model with massless neutrinos. The factor $x_{\nu} \ll 1$ is small for any neutral meson state. Assuming for neutrino masses that $m_{\nu} \sim \sum_{i} m_{\nu_{i}}<0.62 \mathrm{eV}$ [14], where $m_{\nu_{i}}$ is the mass of one of the neutrinos, Eq. (6) yields the branching ratios of $\mathcal{B}_{\mathrm{th}}\left(B_{s}^{0} \rightarrow \nu \bar{\nu}\right)=3.07 \times 10^{-24}$, $\mathcal{B}_{\text {th }}\left(B_{d}^{0} \rightarrow \nu \bar{\nu}\right)=1.24 \times 10^{-25}$, and $\mathcal{B}_{\text {th }}\left(D^{0} \rightarrow \nu \bar{\nu}\right)=1.1 \times$ $10^{-30}$ for $B_{s}, B_{d}$, and $D^{0}$ states, respectively. This led many authors to conclude that invisible decays of heavy meson states are, in fact, background-free modes for searches for new light WILLPs $[15,16]$. Furthermore, these results have led the Particle Data Group (PDG) to list explicit bounds on neutrino masses and references for decays of light mesons into two-neutrino final states [7] from studies of invisible decays.
Here we point out that the $\nu \bar{\nu}$ final state does not constitute a good representation of invisible width of pseudoscalar $\left(0^{-}\right)$mesons in the standard model. In supporting our claim we concentrate on the $B_{q}^{0}$ meson decays, presenting the corresponding results for the $D$ and $K$ states at the end of this article. The same conclusion would also apply to flavor-conserving invisible decays of both heavy (say, $\eta_{c}$ ) and light (say, $\pi^{0}$ ) mesons. Indeed, in the SM the final state that is not detectable in a flavor factory setup contains an arbitrary number of neutrino pairs,

$\mathcal{B}\left(B_{q} \rightarrow \not{E}\right)=\mathcal{B}\left(B_{q} \rightarrow \nu \bar{\nu}\right)+\mathcal{B}\left(B_{q} \rightarrow \nu \bar{\nu} \nu \bar{\nu}\right)+\cdots$

As discussed above [see Eq. (6)], decay to the $\nu \bar{\nu}$ final state is helicity suppressed. The four-neutrino final state, on the other hand, does not suffer from such suppression, so it is expected to have a considerably larger branching ratio. Naively,

$$
\frac{\mathcal{B}\left(B_{q} \rightarrow \nu \bar{\nu} \nu \bar{\nu}\right)}{\mathcal{B}\left(B_{q} \rightarrow \nu \bar{\nu}\right)} \sim \frac{G_{F}^{2} M_{B}^{4}}{16 \pi^{2} x_{\nu}^{2}} \gg 1
$$

In this article, we calculate decays of $B_{q}, D^{0}$, and kaons into a four-neutrino final state. There are only two diagrams that contribute to the decay amplitude when the final state has neutrinos of different flavors. Figure 1(a) shows one such

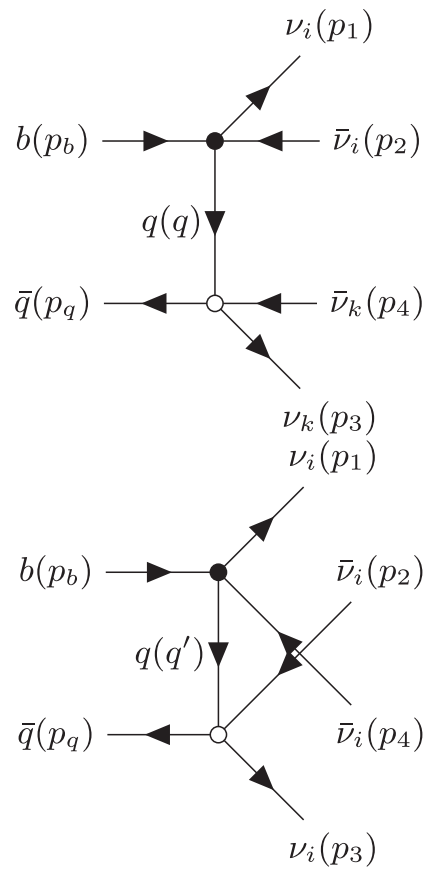

FIG. 1. (a) One of two diagrams for $B_{q} \rightarrow \nu_{i} \bar{\nu}_{i} \nu_{k} \bar{\nu}_{k}$ where $i, k$ refer to different neutrino flavors. (b) Example of an additional diagram that appears in case of identical neutrinos in the final state. Black dots represent flavor-changing vertices, and white dots represent flavor-conserving vertices. 
diagram, while the other can be obtained by replacing the light-quark propagator with a $b$-quark propagator and switching the order of vertices. We also consider the case where the final state neutrinos are flavor identical, in which case additional diagrams appear. Figure 1(b) shows one such additional diagram. The decay amplitude $\mathcal{A}_{q}$ for $B_{q} \rightarrow \bar{\nu} \nu \bar{\nu} \nu$ decay can be written as

$$
\mathcal{A}_{q}=-\frac{G_{F}^{2} \alpha V_{t q}^{*} V_{t b} X\left(x_{t}\right)}{4 \pi \sin ^{2} \theta_{w}} \sum_{i, k} L_{\ell_{i}}^{\mu} L_{\ell_{k}}^{\nu}\left\langle 0\left|\bar{q} \Gamma_{\mu \nu} b\right| B_{q}\right\rangle
$$

where $L_{\ell_{i}}$ are the neutrino currents,

$$
L_{\ell_{i}}^{\mu} \equiv \bar{u}^{\ell_{i}}\left(p_{i}\right) \gamma^{\mu}\left(1-\gamma^{5}\right) v^{\ell_{i}}\left(p_{i+1}\right) .
$$

The effective vertex functions $\Gamma^{\mu \nu}=\sum_{i} \Gamma_{i}^{\mu \nu}$ are given by a combination of vertices and propagators of the light and $b$ quarks, $i=q, b$. A particular example for the diagram Fig. 1(a) is given by

$$
\Gamma_{q}^{\mu \nu}=\gamma^{\nu}\left(c_{V}-c_{A} \gamma^{5}\right) \frac{\left(q+m_{q}\right)}{q^{2}-m_{q}^{2}} \gamma^{\mu}\left(1-\gamma^{5}\right),
$$

where $q=p_{3}+p_{4}-p_{q}, c_{V}^{b}=c_{V}^{s}=-\frac{1}{2}+\frac{2}{3} \sin ^{2} \theta_{w} \equiv c_{V}$ and $c_{A}^{b}=c_{A}^{s}=-\frac{1}{2} \equiv c_{A}$. The other diagrams with the $q$-quark propagator have a similar structure, only differing by the definition of momentum $q$, which follows from momentum conservation. The second set of diagrams is obtained by switching the order of flavor-conserving and flavor-violating vertices.

We adopt a simple model for calculating the transition matrix elements [17],

$$
\left\langle 0\left|\bar{q} \Gamma^{\mu \nu} b\right| B_{s}\right\rangle=\int_{0}^{1} d x \operatorname{Tr}\left[\Gamma^{\mu \nu} \psi_{B}\right],
$$

where $x=p_{b} / P_{B}$ is the momentum fraction of the heavy bottom quark in the $B_{q}$ meson. Here $p_{b}\left(P_{B}\right)$ represents the momentum of the $\mathrm{b}$ quark ( $B_{q}$ meson). The wave function $\psi_{B}$ for the $B_{q}$ meson can be defined as

$$
\psi_{B}=\frac{I_{c}}{\sqrt{6}} \phi_{B}(x) \gamma^{5}\left(P_{B}+M_{B} g_{B}(x)\right),
$$

where $\phi_{B}(x)$ represents the quark distribution amplitude and $I_{C}$ is an identity in color space. We use $g_{B} \approx 1$, which is a good approximation for a heavy meson [17]. The distribution amplitude $\phi_{B}$ contains the $B_{q}$ meson decay constant $\left(f_{B}\right)$ and can be expressed as

$$
\phi_{B}(x)=\frac{f_{B}}{2 \sqrt{3}} \delta(1-x-\xi),
$$

which represents the fact that most of the momentum within the $B_{q}$ meson, $1-\xi$, is carried by a $b$ quark.
Note that $\xi$ is a small parameter, in the case of a $B_{q}$ meson $\xi \approx 0.1$, which allows for expansion of our results in power series in $\xi$. In this article we calculate the leading term in such expansion, neglecting the masses of the light quark and the neutrinos.

The kinematic region for the four-neutrino phase space depends on five independent variables. We follow [18] to define two Mandelstam variables $s_{i j}$ and three helicity angles as our independent variables. Defining $p_{i j}=$ $p_{i}+p_{j}, q_{i j}=p_{i}-p_{j}$ for $\{i, j\}=1-4$, we note that $s_{i j}=$ $p_{i j}^{2}=-q_{i j}^{2}$, and $p_{i j} \cdot q_{i j}=0$. The Mandelstam variables used in this article are $s_{12}=\left(p_{1}+p_{2}\right)^{2}$ and $s_{34}=$ $\left(p_{3}+p_{4}\right)^{2}$, which represent the squared invariant masses of pairs of neutrinos. Then, $p_{12} \cdot p_{34}=\left(M_{B_{q}}^{2}-s_{12}-s_{34}\right) / 2$. The helicity angles are then defined in the centerof-momentum frames for each Mandelstam variable. There are thus two polar angles $\left(\theta_{Y}, \theta_{Z}\right)$ and one azimuthal angle $\left(\theta_{X}\right)$,

$$
\begin{aligned}
p_{12} \cdot q_{34}= & \lambda\left(M_{B}^{2}, s_{12}, s_{34}\right) \cos \theta_{Z} / 2 \\
p_{34} \cdot q_{12}= & \lambda\left(M_{B}^{2}, s_{12}, s_{34}\right) \cos \theta_{Y} / 2 \\
q_{12} \cdot q_{34}= & \left(M_{B_{q}}^{2}-s_{12}-s_{34}\right) \cos \theta_{Y} \cos \theta_{Z} / 2 \\
& +\sqrt{s_{12} s_{34}} \sin \theta_{Y} \sin \theta_{Z} \cos \theta_{X} \\
p_{12}^{\mu} q_{12}^{\nu} p_{34}^{\rho} q_{34}^{\sigma} \epsilon_{\mu \nu \rho \sigma}= & -\lambda\left(M_{B_{q}}^{2}, s_{12}, s_{34}\right) \sqrt{s_{12} s_{34}} \\
& \times \sin \theta_{Y} \sin \theta_{Z} \sin \theta_{X} / 2
\end{aligned}
$$

where $\lambda^{2}(x, y, z)=x^{2}+y^{2}+z^{2}-2 x y-2 y z-2 x z$. In terms of these independent variables the four-body phase space with massless final state particles takes the form

$$
d \Phi=\frac{S}{16 M_{B}(4 \pi)^{6}} d \theta_{X} d \cos \theta_{Y} d \cos \theta_{Z} d s_{34} d s_{12},
$$

where $S=\frac{1}{j !}$ for each group of $j$ identical particles in the final state. While performing the integrals over the four-body phase space we allow the following ranges for the helicity angles, $0 \leq \theta_{X} \leq 2 \pi, 0 \leq \theta_{Y, Z} \leq \pi$, and the Mandelstam variables, $0 \leq s_{34} \leq\left(M_{B_{q}}-\sqrt{s_{12}}\right)^{2}, 0 \leq s_{12} \leq M_{B_{q}}^{2}$. Note that although the integrals over the helicity angles can be performed in any order, the allowed kinematic ranges for the Mandelstam variables were chosen in such a manner that $s_{12}$ is the final variable to be integrated over. As expected, the final result for the decay rate does not depend on this choice of the order of integration, which we perform numerically.

In order to find the total decay rate we consider all three flavors of neutrinos in the final state. There are six different possibilities where the flavors of the two $\nu \bar{\nu}$ pairs in the final state are different, i.e., all four final state particles are distinguishable. In addition, there are three cases where the two $\nu \bar{\nu}$ pairs have the same flavor, and 
hence there are two pairs of identical particles in the final state. We evaluate the rate for each of the two possibilities separately and add them together with appropriate factors (factor of 6 for the former and 3/4 for the latter) to obtain the total decay rate.

To leading order in the expansion in $\xi$ we find that $\Gamma\left(B_{s} \rightarrow \nu \bar{\nu} \nu \bar{\nu}\right)=(2.32 \pm 0.38) \times 10^{-27} \mathrm{GeV}$. Using a $B_{s}$ meson lifetime of $\tau_{B_{s}}=1.509 \mathrm{ps}$ [7], this gives a $\mathcal{B}\left(B_{s} \rightarrow \nu \bar{\nu} \nu \bar{\nu}\right)=(5.48 \pm 0.89) \times 10^{-15}$, which is nine orders of magnitude larger than the SM contribution to $B_{q}^{0}\left(D^{0}\right) \rightarrow \not E$ from the $\nu \bar{\nu}$ final state. Similarly, we find that $\Gamma\left(B_{d} \rightarrow \nu \bar{\nu} \nu \bar{\nu}\right)=(6.54 \pm 1.19) \times 10^{-29} \mathrm{GeV}$. With $\tau_{B_{d}}=1.520 \mathrm{ps}$ from Ref. [7], therefore, we find $\mathcal{B}\left(B_{d} \rightarrow \nu \bar{\nu} \nu \bar{\nu}\right)=(1.51 \pm 0.28) \times 10^{-16}$. For the $D^{0}$ meson decay to four neutrinos, we find that the decay rate is $(4.75 \pm 0.63) \times 10^{-39} \mathrm{GeV}$, and using $\tau_{D^{0}}=410.1 \mathrm{fs}$ from Ref. [7], the branching ratio for the four-body process is $\mathcal{B}\left(D^{0} \rightarrow \nu \bar{\nu} \nu \bar{\nu}\right)=(2.96 \pm 0.39) \times 10^{-27}$. The quoted uncertainties stem from the numerical calculations of phase space integrals. Even though these results are challenging to access experimentally, they are many orders of magnitude larger than the corresponding two-body decays $B_{d}^{0}\left(D^{0}\right) \rightarrow \nu \bar{\nu}$ [8], which also contribute to $B_{q}^{0}\left(D^{0}\right) \rightarrow E$, owing to powerful helicity suppression of the $B_{q}^{0}\left(D^{0}\right) \rightarrow \nu \bar{\nu}$ transitions.

We extend our calculations to also include decays of neutral kaons to four neutrinos. We find that the corresponding decay rates for the $K_{S}^{0}$ and the $K_{L}^{0}$ to be respectively $(4.13 \pm 0.57) \times 10^{-39} \mathrm{GeV}$ and $(3.50 \pm 0.63) \times 10^{-39} \mathrm{GeV}$. Once again using the lifetimes for the neutral kaon initial states $\left(\tau_{K_{S}^{0}}=0.8954 \mathrm{ps}\right.$, and $\tau_{K_{L}^{0}}=0.5116 \mathrm{~ns}$ [7]), we find the branching ratios for the $K_{S}^{0}$ and $K_{L}^{0}$ decays to be (5.62 \pm $0.78) \times 10^{-25}$ and $(2.72 \pm 0.49) \times 10^{-22}$, respectively. In what follows we use experimental data on invisible widths of heavy mesons to constrain properties of dark photons. The dark photons can be properly introduced in the standard model phenomenology by coupling weak isospin field $B_{\mu}$ to a new (dark sector) $U(1)$ vector field $V_{\mu}$ via kinetic mixing [19],

$$
\begin{aligned}
\mathcal{L}= & -\frac{1}{4} W_{3 \mu \nu} W^{3 \mu \nu}-\frac{1}{4} B_{\mu \nu} B^{\mu \nu} \\
& +\frac{\epsilon}{2} B_{\mu \nu} V^{\mu \nu}-\frac{1}{4} V_{\mu \nu} V^{\mu \nu}+\frac{m_{V}^{2}}{2} V_{\mu} V^{\mu},
\end{aligned}
$$

where $\epsilon$ is the kinetic mixing parameter. The field $V_{\mu}$ can acquire mass via a variety of ways. After electroweak symmetry breaking weak isospin field $B_{\mu}$ and the $W_{3 \mu}$ combine to form the $Z$-boson $Z_{\mu}$ and photon $A_{\mu}$ fields with kinetic mixing term $\mathcal{L}=(\epsilon / 2)\left(\cos \theta_{W} F_{\mu \nu}-\sin \theta_{W} Z_{\mu \nu}\right) V^{\mu \nu}$. This term can be eliminated by field redefinition [20],

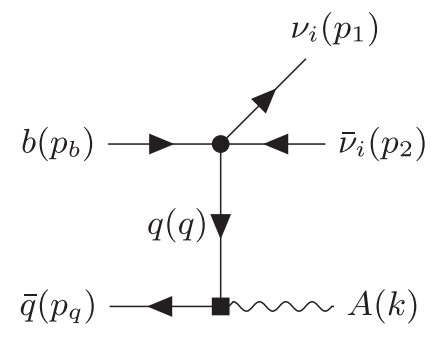

FIG. 2. One of the diagrams leading to $B_{q}^{0} \rightarrow \nu \bar{\nu} V$ transition. Black dot represents flavor-changing vertex [Eq. (1)], while black square represents quark couplings to $V^{\prime}$ [Eq. (19)].

$$
\begin{aligned}
A_{\mu}^{\prime} & =A_{\mu}-\epsilon \cos \theta_{W} V_{\mu}, \\
V_{\mu}^{\prime} & =V_{\mu}+\epsilon \sin \theta_{W} Z_{\mu} .
\end{aligned}
$$

The rotation of Eq. (18) introduces, among other things, a direct coupling between the new dark photon field $V_{\mu}^{\prime}$ and the SM electromagnetic current,

$$
\mathcal{L}=-e \epsilon \cos \theta_{W} J_{\mathrm{em}}^{\mu} V_{\mu}^{\prime},
$$

where $J_{\mathrm{em}}^{\mu}=(2 / 3) \bar{u} \gamma^{\mu} u-(1 / 3) \bar{d} \gamma^{\mu} d+\ldots$ for up and down-type quark fields.

We can now put constraints on the parameters $\epsilon$ and $m_{V}$ from the experimentally constrained invisible $B_{q}^{0}\left(D^{0}\right)$ widths. The lowest order contribution in $\epsilon$ with invisible particles in the final state would be given by the decay $B_{q}^{0}\left(D^{0}\right) \rightarrow V^{\prime} \bar{\nu} \nu$. Similarly to $B_{q}^{0} \rightarrow \gamma \bar{\nu} \nu$ decay $[8,16,21]$ the only contributions that have no helicity suppression are the structure-dependent $V_{\mu}^{\prime}$ emissions given by diagrams of the type pictured in Fig. 2. We use the method of calculating branching ratio for this transition as above; it has been applied to leptonic processes with photon emission $[8,17]$. Unfortunately, current constraints on invisible widths of heavy mesons do not yet permit placing competitive constraints on $\epsilon$. Taking the strongest bound on $B_{d}^{0}$ invisible width and taking the massless limit of $V^{\prime}$ we obtain $|\epsilon|<125$. It is easy to show, however, that the values of $|\epsilon| \sim 3.1 \times 10^{-4}$ could be probed before reaching the " $B_{d}^{0} \rightarrow 4 \nu$ " threshold.

In conclusion, we showed that because of the helicity suppression of the two-neutrino final state, the SM contributions to invisible widths of heavy mesons $\Gamma\left(B_{q}^{0}\left(D^{0}\right) \rightarrow E\right.$ ) are completely dominated by the four-neutrino transitions $B_{q}^{0}\left(D^{0}\right) \rightarrow \nu \bar{\nu} \nu \bar{\nu}$. This implies that invisible decays of pseudoscalar mesons cannot be interpreted as bounds on two-neutrino decay rates and thus cannot be used to set constraints on neutrino masses. The same conclusion also applies to flavor-conserving invisible decays of both heavy and light mesons, rendering the bounds on neutrino masses quoted in the PDG [7] meaningless. Finally, we proved that invisible decays of heavy mesons could be used to probe light 
DM particles [8], but current experimental bounds are insufficient to place meaningful constraints on the properties of dark photons.

This work has been supported in part by the U.S. Department of Energy under Contract No. de-sc0007983. B. B. acknowledges support from Lawrence Technological University faculty seed grant. A. A. P. thanks the Aspen Center for Physics (ACP), where part of this work was performed. ACP is supported by National Science Foundation Grant No. PHY-1607611.
[1] N. Craig, A. Katz, M. Strassler, and R. Sundrum, J. High Energy Phys. 07 (2015) 105.

[2] P. Meade, S. Nussinov, M. Papucci, and T. Volansky, J. High Energy Phys. 06 (2010) 029.

[3] G. Aad et al. (ATLAS Collaboration), Phys. Rev. Lett. 108, 251801 (2012).

[4] G. Aad et al. (ATLAS Collaboration), Phys. Rev. D 92, 012010 (2015).

[5] R. Aaij et al. (LHCb Collaboration), Eur. Phys. J. C 77, 812 (2017).

[6] R. Essig et al., arXiv:1311.0029.

[7] C. Patrignani et al. (Particle Data Group), Chin. Phys. C 40, 100001 (2016); M. Tanabashi et al. (Particle Data Group), Phys. Rev. D 98, 030001 (2018).

[8] A. Badin and A. A. Petrov, Phys. Rev. D 82, 034005 (2010).

[9] C. L. Hsu et al. (Belle Collaboration), Phys. Rev. D 86, 032002 (2012).

[10] J. P. Lees et al. (BABAR Collaboration), Phys. Rev. D 86, 051105 (2012).
[11] Y.-T. Lai et al. (Belle Collaboration), Phys. Rev. D 95, 011102 (2017).

[12] G. Buchalla and A. Buras, Nucl. Phys. B400, 225 (1993).

[13] T. Inami and C. S. Lim, Prog. Theor. Phys. 65, 297 (1981); 65, 1772(E) (1981).

[14] A. Goobar, S. Hannestad, E. Mortsell, and H. Tu, J. Cosmol. Astropart. Phys. 06 (2006) 019.

[15] S. de Boer, Proc. Sci., BEAUTY2018 (2018) 022 [arXiv: 1807.05845].

[16] C. D. Lu and D. X. Zhang, Phys. Lett. B 381, 348 (1996).

[17] Y. G. Aditya, K. J. Healey, and A. A. Petrov, Phys. Lett. B 710, 118 (2012).

[18] W. Altmannshofer, P. Ball, A. Bharucha, A. J. Buras, D. M. Straub, and M. Wick, J. High Energy Phys. 01 (2009) 019.

[19] B. Holdom, Phys. Lett. 166B, 196 (1986).

[20] M. Baumgart, C. Cheung, J. T. Ruderman, L. T. Wang, and I. Yavin, J. High Energy Phys. 04 (2009) 014.

[21] T. M. Aliev, A. Ozpineci, and M. Savci, Phys. Lett. B 393, 143 (1997). 